\title{
An Adaptive Traffic Signaling for Roundabout with Four-Approach Intersections Based on Fuzzy Logic
}

\author{
Maged M. M. Fahmy \\ Computer Department, College of Applied Studies, King Faisal University, Saudi Arabia
}

\begin{abstract}
This paper presents an adaptive traffic signaling method based on fuzzy logic for roundabout with four-approach intersection. The process of whether to extend or terminate current signal phase and select the sequence of next phases is determined using fuzzy logic. The proposed method can replace an experienced traffic policeman organizing traffic at roundabout intersections.
\end{abstract}

Keywords: fuzzy logic, traffic signaling, roundabout with four approach intersections, expert systems.

\section{Introduction}

Roundabouts are widely used in Gulf area for organizing traffic at road intersections. Big traffic jams, especially at the roundabout intersections, are happening at peak hours. Since there is no traffic signal to organize the traffic at these areas, a traffic policeman has to stand there for long hours in the very hot weather (in summer in Gulf area) to organize the traffic. Off-line traffic signaling cannot solve the problem because it would not take into consideration the rush hour traffic, or that one direction of a fourway intersected roundabout may not have much traffic. A lane may be really busy because it is an alternate route due to an accident in the other route. It would be too difficult for a person to reprogram them to be robust enough to handle all possible scenarios effectively. Therefore, adaptive traffic signaling would solve the problem. This research paper aims at using intelligent traffic signaling system, based on fuzzy logic, that can organize the vehicles entering a roundabout according to both traffic needs and vehicles priority in those areas.

This system will perform standard function of controlling traffic based on how long cars have been waiting at a stop signal and the traffic quantity there. The system would have a memory and would remember for how long the traffic was held at that traffic signal and it would be able to handle congestion more efficiently.

Traffic signal is an essential element used to manage the transportation network. Basically, there are two types of conventional traffic signal control systems in use. First type is Off-line traffic signaling that uses a preset cycle time to change the lights. The second type of control combines preset cycle time with proximity sensors which can activate a change in the cycle time or the lights. Roads with less cars may not need a regular cycle of green lights. Proximity sensors will activate a change in the light when cars are present. This type of control depends on having some prior knowledge of traffic flow patterns at the intersection so that signal cycle times and placement of proximity sensors may be customized for the intersection. Recently, a major research has been focused on applications of artificial intelligence techniques such as expert systems, fuzzy logic, neural networks and genetic algorithms for traffic signal control [1-6].

Fuzzy traffic signal control is an alternative to conventional traffic lights control that can be used for a wider array of traffic patterns at a roundabout. A fuzzy logic controlled traffic light uses sensors that count cars instead of proximity sensors which only indicate the presence of cars. This provides the controller with traffic densities in the lanes and allows better assessment of changing traffic patterns. As 
the traffic distributions fluctuate, the fuzzy controller can change the signal light accordingly.

The main reason why fuzzy set theory is a suitable approach to adaptive traffic signal control is the nature of uncertainties in signal control. Decisions are made based on imprecise information. The consequences of decisions are not well known. The objectives have no clear priorities. It has been known through various experiments and applications that fuzzy control is well suited when the control involves these kinds of uncertainties and human perception. Through the application of neural linguistic variables and the heuristic of human reasoning, fuzzy logic can utilize imprecise or incomplete information. Fuzzy logic adaptive traffic signaling control (FLATSC) uses rule-based logic to easily incorporate traffic policeman expertise about traffic signaling. In this research, this system is built and utilized at roundabout junctions to adapt traffic signals there.

\section{Design Issues for FLATSC}

General structure of a fuzzy traffic control system for roundabout with four-approach intersections is illustrated in Figure 1. There are two sensors placed on each incoming roundabout lane. The first sensor (downstream sensor) behind the traffic lights counts the cars passing the traffic lights, and the second sensor (upstream sensor), which is located behind the first sensor, counts the cars coming to the roundabout at distance $\mathrm{D}$ from the lights. The amount of cars for a roundabout lane is determined by the difference of the readings between the two sensors. This is in contrast to conventional control systems that place a proximity sensor at the front of each traffic lights and can only sense the presence of a car waiting at the junction, not the amount of cars waiting at the roundabout lane. The distance between the two sensors D is determined accordingly, following the traffic flow pattern at that particular roundabout. The fuzzy logic controller is responsible for determining which approach has the highest priority in order to give it the green light. This is in accordance with overall traffic conditions at that roundabout. The state machine controls the sequence of state that the fuzzy traffic controller should cycle through. There is one default state that takes place when no incoming traffic is detected. This default state corresponds to the green light time for a specific approach, usually to the main approach. A green light can be skipped if there is no vehicle queue for the corresponding approach.

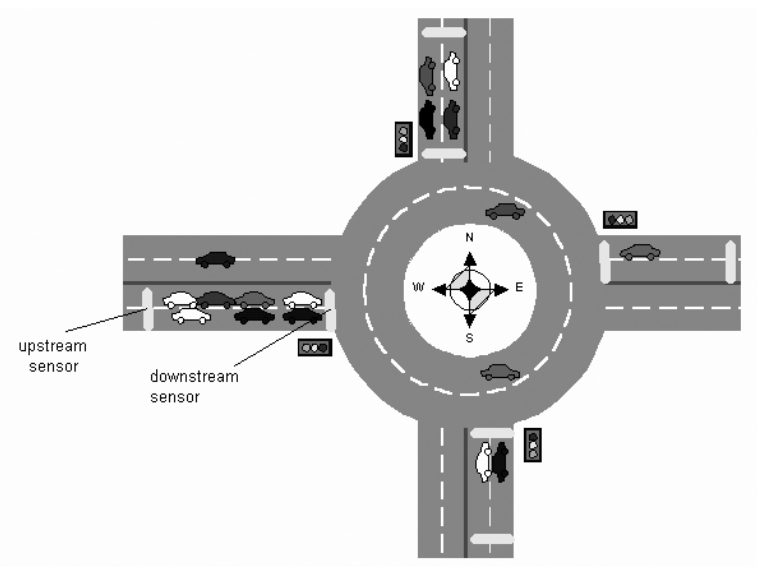

Fig. 1. Structure of fuzzy traffic control system.

As shown in Figure 1, the roundabout has four approaches: Western northern approach, Eastern northern approach, Eastern approach, and Western southern approach. The incoming approaches are numbered according to their importance and priority in the specified location.

\subsection{Design Criteria and Constraints}

A fuzzy logic controller was designed for a roundabout with four intersected roads as shown in Figure 2. The controller has two fuzzy input variables: the quantity of the traffic waiting on the arrival side and the traffic waiting time (according to up-stream and down-stream sensors). The output fuzzy variable would be the priority degree given for each roundabout approach. In the development of the fuzzy traffic lights control system the following assumptions are made:

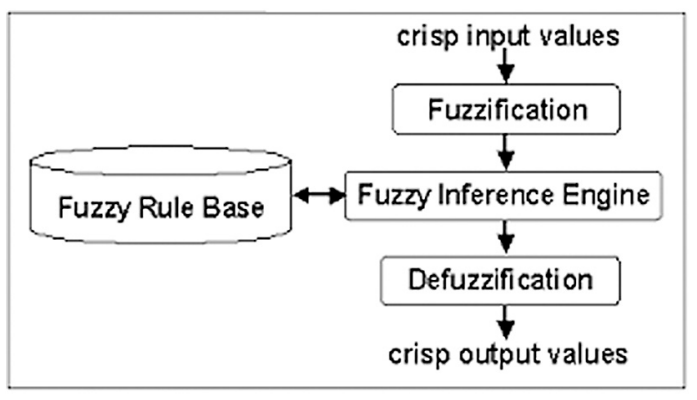

Fig. 2. Fuzzy Expert System Model. 
- The junction is four intersected roads in a roundabout, with traffic coming from four different directions.

- When traffic from the north moves, traffic from the other approaches stops, and so on.

- The north and south lanes are assumed to be the main approaches.

\section{Collecting Input Data to ITCS}

To determine the traffic quantity in a given track section, vehicles entering and leaving the section are counted. For this purpose, a sensor must be able to detect the travel direction of a passing wheel or axle in order to tell whether to count up or down. Also, the sensor must be able to correctly handle situations where a wheel is entering its detection zone from one direction, stopping and leaving it in the same direction.

This is normally achieved by using a pair of sensors (Fiber optic wheel sensors) with overlapping detection zones. Traffic quantity and waiting time are calculated using the terminology in Table 1 and the equations 1 and 2 following to it.

\begin{tabular}{|l|l|c|}
\hline Input & Detector Type & Extension \\
\hline \hline $\begin{array}{l}\text { Upstream } \\
\text { Direction }\end{array}$ & $\begin{array}{l}\text { Upstream } \\
\text { Sensor }\end{array}$ & $\mathrm{D}_{\text {in }}$ \\
\hline $\begin{array}{l}\text { Downstream } \\
\text { Direction }\end{array}$ & $\begin{array}{l}\text { Downstream } \\
\text { Sensor }\end{array}$ & $\mathrm{D}_{\text {out }}$ \\
\hline $\begin{array}{l}\text { Upstream } \\
\text { Quantity }\end{array}$ & $\begin{array}{l}\text { Upstream } \\
\text { Sensor }\end{array}$ & $\mathrm{Q}_{\text {in }}$ \\
\hline $\begin{array}{l}\text { Downstream } \\
\text { Quantity }\end{array}$ & $\begin{array}{l}\text { Downstream } \\
\text { Sensor }\end{array}$ & $\mathrm{Q}_{\text {out }}$ \\
\hline $\begin{array}{l}\text { Upstream } \\
\text { Time }\end{array}$ & $\begin{array}{l}\text { Upstream } \\
\text { Sensor }\end{array}$ & $\mathrm{T}_{\text {in }}$ \\
\hline $\begin{array}{l}\text { Downstream } \\
\text { Time }\end{array}$ & $\begin{array}{l}\text { Downstream } \\
\text { Sensor }\end{array}$ & $\mathrm{T}_{\text {out }}$ \\
\hline
\end{tabular}

Table 1. Description of Algorithm Inputs.

The Upstream Direction and Downstream Direction are used to indicate the direction of the incoming and outgoing vehicles. The Upstream Quantity and Downstream Quantity are used to determine the number of waiting vehicles for a specific approach using the equation:

$$
\mathrm{Q}_{\text {final }}=\mathrm{Q}_{\text {out }}-\mathrm{Q}_{\text {in }}
$$

The Upstream Time and Downstream Time are used to determine vehicles waiting time using the equation:

$$
\mathrm{T}_{\text {waiting }}=\mathrm{T}_{\text {out }}-\mathrm{T}_{\text {in }}
$$

\section{Fuzzy Logic Traffic Signal Controller}

Fuzzy logic traffic signal controller consists of a fuzzy rule base, fuzzification module, inference engine, and defuzzification module (Figure 2). The inputs to the controller are calculated from loop detector data sent from the road sensors to the online embedded computer system. For each lane sensors, there are two inputs inserted to the fuzzy algorithm (waiting time and traffic quantity). The fuzzification module pre-processes the input values submitted to the fuzzy expert system. The inference engine uses the results of the fuzzification module and accesses the fuzzy rules in the fuzzy rule base to infer what intermediate and output values to produce (priority degree in this research). The defuzzification module provides crisp value of the final output of the fuzzy expert system (green light time). FuzzyTECH 5.52 package is used in simulating this controller.

\subsection{Fuzzy Input and Output Variables}

\begin{tabular}{|l|c||l|c||l|c|}
\hline $\begin{array}{l}\text { Traffic } \\
\text { Quantity }\end{array}$ & \multicolumn{2}{l||l|l|}{$\begin{array}{l}\text { Waiting } \\
\text { Time }\end{array}$} & \multicolumn{2}{l|}{$\begin{array}{l}\text { Priority } \\
\text { Degree }\end{array}$} \\
\hline \hline Low & L & Low & L & Low & L \\
\hline Medium & M & Medium & M & Medium & M \\
\hline High & H & High & H & High & H \\
\hline
\end{tabular}

Table 2. Fuzzy variables of traffic quantity, waiting time, and priority degree of the traffic light control system.

Fuzzy variables of the traffic light control system are: traffic quantity and waiting time as inputs, and priority degree as output. They are shown in Table 2., where the right hand notations are used to shorten these variables. 


\subsection{Membership Functions}

For the traffic lights controller, there are three membership functions for each of the input and output fuzzy variables. The membership function for each fuzzy linguistic variable, together with its graphical representation, is illustrated in Figures 3, 4, and 5. It can be observed that the $y$-axis is the degree of the membership of each of the fuzzy variables. For the input fuzzy variables, the universe of discourse (the X-axis) is the quantized sensor signal that sensed the quantity and the waiting time of vehicles. For the output fuzzy variable, the universe of discourse is the priority degree of the given approach. From Figure 3, and according to the traffic quantity fuzzy set (Equation 3), it can be observed that six cars in approach four have been assigned as "M" fuzzy subset, and two cars in the third approach, four cars in the first approach and one car in the second approach have been assigned as "L" fuzzy subset. According to the Waiting Time fuzzy set, assume that the fourth approach has been assigned as " $\mathrm{H}$ " waiting time and the first approach as "M", second and third approaches as "L", since they have waited for short time. Using Fuzzy Rules, the output fuzzy set function will assign approach four as " $H$ " priority degree and the rest of approaches as "L" fuzzy set. The traffic signal of the fourth approach will win green light while the rest of approaches are assigned red. While the green light is on, the online system will continue detecting the incoming and outgoing cars to determine their number and waiting time, in order to produce a robust online system with no weaknesses. Since the other three approaches have the same degree of output fuzzy subset which is "L", the priority of getting the green light will be determined separately according to their priority which will be determined in the next phase when approaches are competing again to win green light. The process continues. The configuration of these membership functions is done according to human expert observations and the environment. Membership function of input fuzzy set are: high (TQ) that determines how much the traffic is crowded (Equation 3), high (WT) that determines how long the waiting time is (Equation 4), and high (PD) that determines the priority degree of a given lane (Equation 5):

$$
\operatorname{high}(\mathrm{TQ})=\left\{\begin{array}{cl}
0, & \text { if } \mathrm{TQ}<5, \\
\frac{(\mathrm{TQ}-5)}{10}, & \text { if } 5 \leq \mathrm{TQ} \leq 15, \\
1, & \text { if } \mathrm{TQ}>15
\end{array}\right.
$$

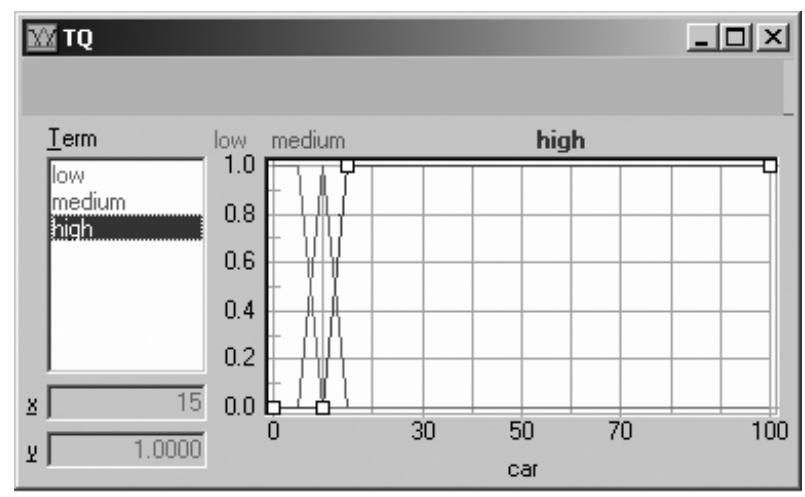

Fig. 3. Traffic Quantity (TQ) - the first input of the fuzzy logic controller.

$\operatorname{high}(\mathrm{WT})=\left\{\begin{array}{cl}0, & \text { if } \mathrm{WT}<20, \\ \frac{(\mathrm{WT}-20)}{40}, & \text { if } 20 \leq \mathrm{WT} \leq 60, \\ 1, & \text { if } \mathrm{WT}>60\end{array}\right.$

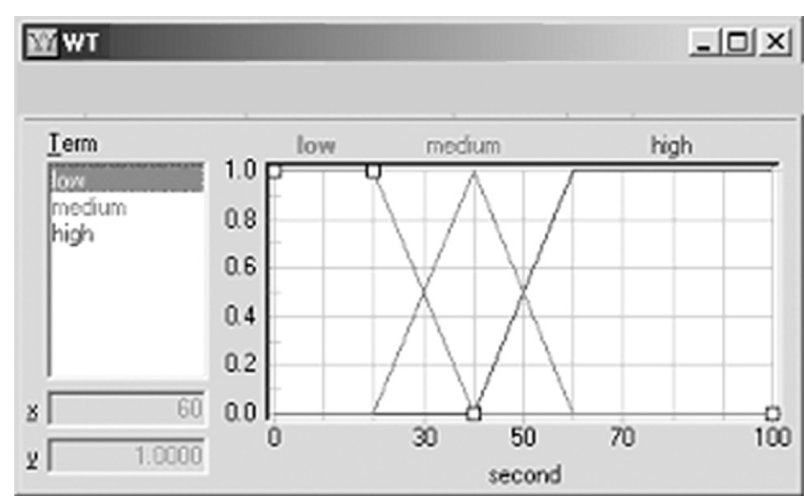

Fig. 4. Waiting Time (WT) - the second input of the fuzzy logic controller.

high $(\mathrm{PD})=\left\{\begin{array}{cl}0, & \text { if } \mathrm{PD}<0.25 \\ \frac{(\mathrm{PD}-0.25)}{0.5}, & \text { if } 0.25 \leq \mathrm{PD} \leq 0.75 \\ 1, & \text { if } \mathrm{PD}>0.75\end{array}\right.$ 


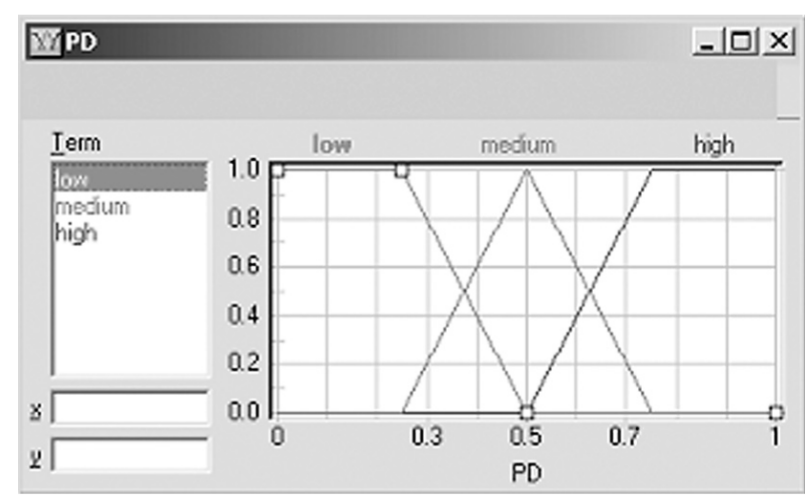

Fig. 5. Priority Degree - the output (PD) of the fuzzy logic controller.

However, the width and the center of membership functions of these fuzzy subsets can be easily changed and configured according to different traffic situations and conditions. For example, if the junction is too congested, the number of cars in the fuzzy subset "high TQ" can be increased. On the other hand, for a less congested junction the width of the membership functions can be reduced. It can be observed that in fuzzy logic control the transition from one fuzzy subset to another should be a smooth transition, which needs to overlap these fuzzy subsets. If there were no overlapping in the fuzzy subsets then the control action would resemble bivalent control (step-like action).

\subsection{Fuzzy Rule Base}

The inference mechanism in the fuzzy logic controller resembles that of the human reasoning process. This is where fuzzy logic technology is associated with artificial intelligence. Humans unconsciously use rules in implementing their actions. For example, a traffic policeman manning a junction would use his expert opinion in controlling the traffic more or less in the following way:

IF the traffic quantity is Very High

AND IF the traffic waits for Long time

THEN allow movement of traffic for Long Time

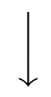

\section{IF TQ is $\mathrm{H}$ AND WT is $\mathrm{H}$ THEN PD is $\mathrm{H}$}

Another opinion would be:
IF the traffic quantity is Normal AND IF the traffic waits Too Much THEN allow movement for Long Time,<smiles>C1=CC=C1</smiles>

\section{IF TQ is M AND WT is $\mathrm{H}$ THEN PD is $\mathrm{H}$}

where: TQ is Traffic Quantity, WT is Waiting Time, and PD is Priority Degree fuzzy sets.

TQ and WT are the antecedents of the specified approach and PD is the consequent. Such rules can be easily developed according to the conditions of the traffic at the junction. Table 3 shows a compact way of writing these rules as a matrix.

\begin{tabular}{|c||c|c|c|c|}
\hline \multicolumn{5}{|c|}{ TQ } \\
\hline \hline \multirow{4}{*}{ WT } & & L & M & H \\
\cline { 2 - 5 } & L & L & L & M \\
\cline { 2 - 5 } & M & L & M & H \\
\cline { 2 - 6 } & H & M & H & H \\
\hline
\end{tabular}

Table 3. Fuzzy rules of Traffic Signal Control.

The size of this matrix, or the number of rules, is equal to the number of input combinations derived from the number of membership functions per input. If in the traffic control system there are two inputs, each having three membership functions, then the number of rules would equal nine as the following:

rule 1: if TQ is low and WT low is then PD is low

rule 2: if TQ is low and $\mathrm{WT}$ is medium then $\mathrm{PD}$ is low

rule 3: if TQ is low and WT is high then PD is medium

rule 4: if TQ is medium and $\mathrm{WT}$ is low then PD is low

rule 5: if TQ is medium and WT is medium then PD is medium

rule 6: if TQ is medium and $\mathrm{WT}$ is high then $\mathrm{PD}$ is high

rule 7: if TQ is high and WT is low then PD is medium

rule 8: if TQ is high and WT is medium then $\mathrm{PD}$ is high

rule 9: if TQ is high and $\mathrm{WT}$ is high then $\mathrm{PD}$ is high 
In many applications it is not necessary to fill up all the rules in the matrix bank, however, for this application it is necessary (Figure 6).

\begin{tabular}{|l|l|l|l|l|}
\hline \multirow{2}{*}{ I } & IF & WT & THEN & \\
& TQ & DoS & PD \\
\hline 1 & low & low & 1.00 & low \\
\hline 2 & low & medium & 1.00 & low \\
\hline 3 & low & high & 1.00 & medium \\
\hline 4 & medium & low & 1.00 & low \\
\hline 5 & medium & medium & 1.00 & medium \\
\hline 6 & medium & high & 1.00 & high \\
\hline 7 & high & low & 1.00 & medium \\
\hline 8 & high & medium & 1.00 & high \\
\hline 9 & high & high & 1.00 & high \\
\hline & & & & \\
\hline
\end{tabular}

Fig. 6. Fuzzy rules of Traffic signal control.

Since our proposed system is designed to solve the traffic problem in the roundabouts area, the crisp output that represents the priority degree of each of the four incoming lanes must be compared at the end to find the winning approach that has the highest priority degree.

\subsection{Inference Process}

The following are the input membership functions and rules defined for our proposed traffic control system:

$\operatorname{high}(\mathrm{TQ})=\left\{\begin{array}{cl}0, & \text { if } \mathrm{TQ}<5, \\ \frac{(\mathrm{TQ}-5)}{10}, & \text { if } 5 \leq \mathrm{TQ} \leq 15, \\ 1, & \text { if } \mathrm{TQ}>15\end{array}\right.$
$\operatorname{high}(\mathrm{WT})=\left\{\begin{array}{cl}0, & \text { if } \mathrm{WT}<20, \\ \frac{(\mathrm{WT}-20)}{40}, & \text { if } 20 \leq \mathrm{WT} \leq 60, \\ 1, & \text { if } \mathrm{WT}>60\end{array}\right.$

$\begin{array}{cl}\text { medium } \\ (\mathrm{TQ})\end{array}=\left\{\begin{array}{cl}0, & \text { if high } \mathrm{TQ}=0 \\ \text { or hight } \mathrm{TQ}=1, \\ 0.2 *(\mathrm{TQ}-5), & \text { if } 5<\mathrm{TQ}<10, \\ 1-(0.2 *(\mathrm{TQ}-5)), & \text { if } 10<\mathrm{TQ}<15, \\ 1, & \text { if high } \mathrm{TQ}=0.5\end{array}\right.$

$$
\underset{(\mathrm{WT})}{\text { medium }}=\left\{\begin{array}{cl}
0, & \text { if high } \mathrm{WT}=0 \\
& \text { or hight } \mathrm{WT}=1, \\
0.05 *(\mathrm{WT}-20), & \text { if } 20<\mathrm{WT}<40, \\
1-(0.05 *(\mathrm{WT}-40)), & \text { if } 40<\mathrm{WT}<60 \\
1, & \text { if high } \mathrm{WT}=0.5
\end{array}\right.
$$

$\operatorname{low}(\mathrm{TQ})=1-\operatorname{high}(\mathrm{TQ})$

$\operatorname{low}(\mathrm{WT})=1-\operatorname{high}(\mathrm{WT})$

The inference process uses several rules simultaneously. This process is a combination of four sub-processes: fuzzification, inference, composition, and defuzzification. The defuzzification sub-process is an optional one.

\subsubsection{Fuzzification}

Membership functions, defined on the input variables, are applied to their actual values to determine the degree of truth for each rule premise. The most popular fuzzy implementation of the AND operator is the minimum function. This function takes two values or operations and returns the lowest one. For example:

$$
\operatorname{AND}(\mathrm{A}, \mathrm{B})=\operatorname{Minimum}(\operatorname{truth}(\mathrm{A}), \operatorname{truth}(\mathrm{B}))
$$

To examine the fuzzification process on our nine rules, some test values are needed for the input variables TQ and WT. Each rule will then be evaluated on these variables using the fuzzy AND operator to produce another value. If this value is non-zero then the rule is said to 'fire'. Notice that the alpha of each rule is not the value assigned to the output variable PD. It is simply used to represent the degree of truth of the premise of each rule.

In case of the values 3.2 and 0 for the input variables $\mathrm{X}$ and $\mathrm{Y}$ respectively, the nine rules are evaluated as the following:

rule 1: if TQ is low AND

$$
\mathrm{WT} \text { is low then PD }=\text { low }
$$

low(TQ) AND low(WT)

low(30) AND low(54)

0.00 AND 0.15

$\operatorname{minimum}(0.00,0.15)=0.00$

Since the alpha of rule 1 upon the input values of 30 and 54 is 0.00 , rule 1 does not 'fire'. 
The same operation is done up to rule 9:

rule 9: if TQ is high AND

WT is high then PD = high

high (TQ) AND high(WT)

high (30) AND high(54)

1.00 AND 0.85

minimum $(1.00,0.85)=0.85$

Since the alpha of rule 9 upon the input values of 30 and 54 is 0.85 , rule 9 is said to 'fire'.

The degree of truth for a rule's premise is sometimes referred to as its alpha. If a rule's premise has a nonzero degree of truth then the rule is said to 'fire'. Table 4 shows values for input data collected using the road sensors and corresponding values results from applying membership functions. Table 5 shows the degree of truth for rules 1 to 9 (Fuzzification process).

\subsubsection{Inference}

The inference process is concerned with applying the truth of each rule (its alpha) to the concluding part. Thus, an entire fuzzy subset is assigned to the output variable. Usually only MIN or PRODUCT are used as inference rules. In

\begin{tabular}{|c|c|c|c|c|c|c|c|}
\hline TQ & WT & L(TQ $)$ & $\mathrm{L}(\mathrm{WT})$ & $\mathrm{M}(\mathrm{TQ})$ & $\mathrm{M}(\mathrm{WT})$ & $\mathrm{H}(\mathrm{TQ})$ & $\mathrm{H}(\mathrm{WT})$ \\
\hline \hline 30 & 54 & 0.00 & 0.15 & 0.00 & 0.30 & 1.00 & 0.85 \\
\hline 9 & 60 & 0.60 & 0.00 & 0.80 & 0.00 & 0.40 & 1.00 \\
\hline 6 & 41 & 0.90 & 0.47 & 0.20 & 0.95 & 0.10 & 0.53 \\
\hline 10 & 18 & 0.50 & 1.00 & 1.00 & 0.00 & 0.50 & 0.00 \\
\hline 13 & 30 & 0.20 & 0.75 & 0.40 & 0.50 & 0.80 & 0.25 \\
\hline 2 & 4 & 1.00 & 1.00 & 0.00 & 0.00 & 0.00 & 0.00 \\
\hline 42 & 73 & 0.00 & 0.00 & 0.00 & 0.00 & 1.00 & 1.00 \\
\hline 8 & 40 & 0.70 & 0.50 & 0.60 & 1.00 & 0.30 & 0.50 \\
\hline 2 & 15 & 1.00 & 1.00 & 0.00 & 0.00 & 0.00 & 0.00 \\
\hline 16 & 22 & 0.00 & 0.95 & 0.00 & 0.10 & 1.00 & 0.05 \\
\hline
\end{tabular}

Table 4. Input data collected using the road sensors and corresponding values results from applying membership functions. L, M, H stands for low, medium and high.

\begin{tabular}{|c|c|c|c|c|c|c|c|c|}
\hline alpha1 & alpha2 & alpha3 & alpha4 & alpha5 & alpha6 & alpha7 & alpha8 & alpha9 \\
\hline \hline 0.00 & 0.00 & 0.00 & 0.00 & 0.00 & 0.00 & 0.15 & 0.30 & 0.85 \\
\hline 0.00 & 0.00 & 0.60 & 0.00 & 0.00 & 0.80 & 0.00 & 0.00 & 0.40 \\
\hline 0.47 & 0.90 & 0.53 & 0.20 & 0.20 & 0.20 & 0.10 & 0.10 & 0.10 \\
\hline 0.50 & 0.00 & 0.00 & 1.00 & 0.00 & 0.00 & 0.50 & 0.00 & 0.00 \\
\hline 0.20 & 0.20 & 0.20 & 0.40 & 0.40 & 0.25 & 0.75 & 0.50 & 0.25 \\
\hline 1.00 & 0.00 & 0.00 & 0.00 & 0.00 & 0.00 & 0.00 & 0.00 & 0.00 \\
\hline 0.00 & 0.00 & 0.00 & 0.00 & 0.00 & 0.00 & 0.00 & 0.00 & 1.00 \\
\hline 0.50 & 0.70 & 0.50 & 0.50 & 0.60 & 0.50 & 0.30 & 0.30 & 0.30 \\
\hline 1.00 & 0.00 & 0.00 & 0.00 & 0.00 & 0.00 & 0.00 & 0.00 & 0.00 \\
\hline 0.00 & 0.00 & 0.00 & 0.00 & 0.00 & 0.00 & 0.95 & 0.10 & 0.05 \\
\hline
\end{tabular}

Table 5. Fuzzification process. 
MIN inferencing, the output membership function is clipped off at a height corresponding to the rule premise's computed degree of truth (fuzzy logic AND). In PRODUCT inferencing, the output membership function is scaled by the rule premise's computed degree of truth. When the input values 30 and 54 were inserted for the variables TQ and WT, the alphas of the nine rules were $0.00,0.00,0.00,0.00,0.00,0.00$, $0.15,0.30$ and 0.85 respectively. Since only the seventh, eighth and ninth rules produced nonzero alphas, only these three rules will be considered. The conclusion part of rule 7 assigned the fuzzy membership function 'medium' to the output variable PD. Since the degree of truth of rule 7 when supplied with 30 and 54 was 0.15 , the output variable PD is assigned the following fuzzy subset when using MIN inferencing:

$\begin{array}{cl}\text { rule7 } \\ (\mathrm{PD})\end{array}=\left\{\begin{array}{cl}0, & \text { if } \mathrm{PD} \leq 0.25 \\ & \text { or } \mathrm{PD} \geq 0.75, \\ 0.15, & \text { if } \mathrm{PD}=0.50 \\ 0.6 *(\mathrm{PD}-0.25), & \text { if } 0.25<\mathrm{PD}<0.50, \\ 0.15-(0.6 *(\mathrm{PD}-0.50)), & \text { if } 0.50<\mathrm{PD}<0.75,\end{array}\right.$

The membership graph is therefore (Figure 7):

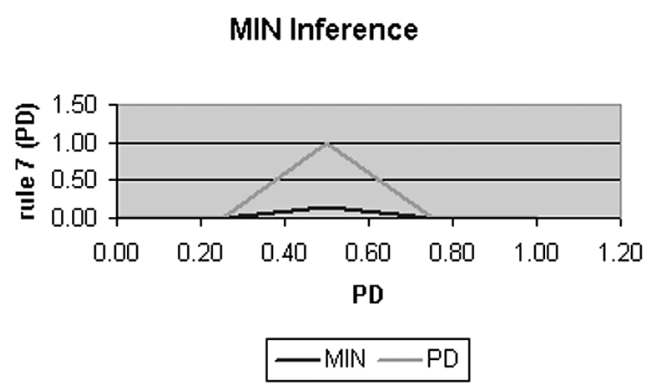

Fig. 7. The result using MIN inference with rule7 - TQ: 30 and WT: 54.

Note how all values for 'rule7(PD)' greater than 0.15 have been clipped off the high membership function graph.

The conclusion part of rule 8 assigned the fuzzy membership function 'high' to the output variable PD. Since the degree of truth of rule 8 when supplied with 30 and 54 was 0.30 , the output variable PD is assigned the following fuzzy subset when using MIN inferencing:

$$
\text { rule8 }(\mathrm{PD})=\left\{\begin{array}{cl}
0, & \text { if } \mathrm{PD} \leq 0.50 \\
& \text { becouse high } \mathrm{PD} \\
& \text { is applied in rule } 8 \\
6 *(\mathrm{PD}-0.50), & \text { if } 0.50 \leq \mathrm{PD} \leq 0.57 \\
& \text { if } \mathrm{PD} \geq 0.57 \\
0.30, \quad & \text { becouse high }(\mathrm{PD}) \\
& =0.3,
\end{array}\right.
$$

The membership graph is seen in Figure 8.

MIN Inference

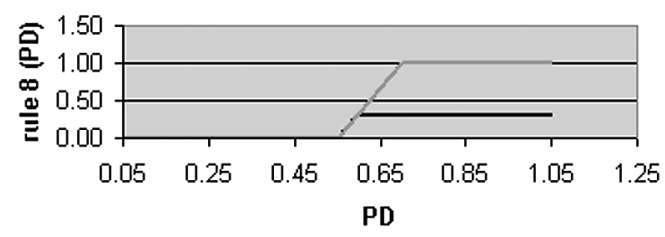

$-\mathrm{M} I \mathrm{~N}-\mathrm{PD}$

Fig. 8. The result using MIN inference with rule8 - TQ: 30 and WT: 54.

Notice how all values for 'rule8(PD)' greater than 0.30 have been clipped off the high membership function graph.

The conclusion part of rule 9 assigned the fuzzy membership function ' high' to the output variable PD. Since the degree of truth of rule 9 when supplied with 30 and 54 was 0.85 , the output variable PD is assigned the following fuzzy subset when using MIN inferencing:

rule9 $(\mathrm{PD})=\left\{\begin{array}{cl}0, & \text { if } \mathrm{PD} \leq 0.50 \\ 2.428 *(\mathrm{PD}-0.5), & \text { if } 0.5 \leq \mathrm{PD} \leq 0.85, \\ 0.85, & \text { if } \mathrm{PD} \geq 0.85\end{array}\right.$

The membership graph is seen in Figure 9.

MIN Inference

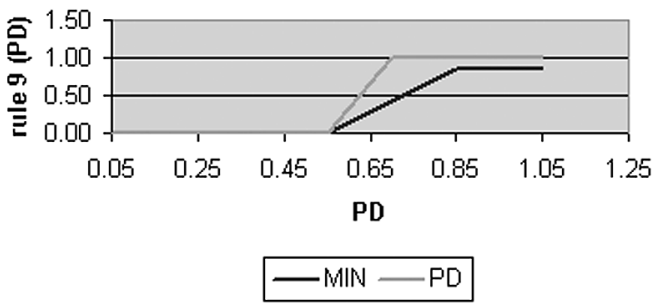

Fig. 9. The result using MIN inference with rule9-TQ: 30 and WT: 54. 
Note how all values for 'rule9(PD)' greater than 0.85 have been clipped off the high membership function graph.

MIN inferencing can also be applied to those rules that did not 'fire', such as rules 1, 2, 3, 4, 5 , and 6 in our example. However, as the result would be a graph clipped off at 0.0 , there is little point. As already discussed, another inferencing process called PRODUCT can also be used for assigning fuzzy subsets. With this inferencing process, the output membership function is scaled by the alpha of the rule.

The conclusion part of rule 7 assigned the fuzzy membership function 'medium' to the output variable PD. Since the degree of truth of rule 7 when supplied with 30 and 54 was 0.15 , the output variable PD is assigned the following fuzzy subset when using PRODUCT inferencing:

$$
\begin{gathered}
\text { rule7.a(PD) }=0.6 *(\mathrm{PD}-0.25) \\
\text { if } \quad 0.25<\mathrm{PD}<0.50 \\
\text { rule7.b(PD) }=0.15-(0.6 *(\mathrm{PD}-0.50)) \\
\text { if } \quad 0.50<\mathrm{PD}<0.75
\end{gathered}
$$

The membership graph is seen in Figure 10.

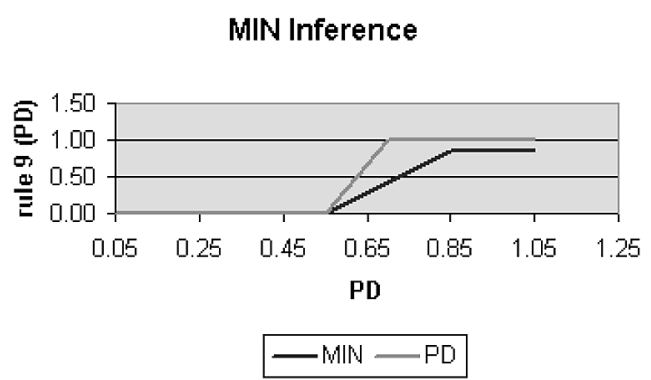

Fig. 10. The result using PRODUCT inference with rule7 - TQ: 30 and WT: 54

The conclusion part of rule 8 assigned the fuzzy membership function 'high' to the output variable PD. Since the degree of truth of rule 8 when supplied with 30 and 54 was 0.30 , the output variable PD is assigned the following fuzzy subset when using PRODUCT inferencing:

$$
\operatorname{rule} 8(\mathrm{PD})=0.6 *(\mathrm{PD}-0.5)
$$

The membership graph is seen in Figure 11.

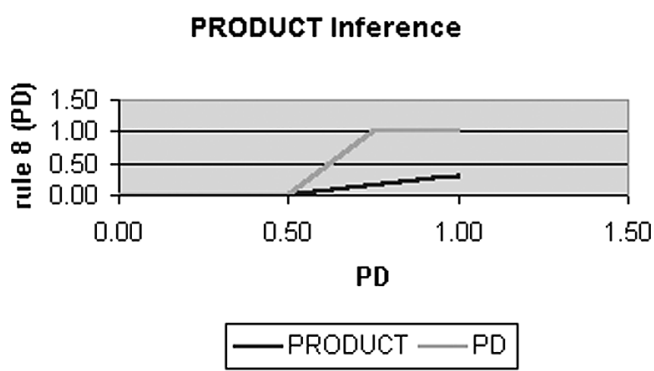

Fig. 11. The result using PRODUCT inference with rule8 - TQ: 30 and WT: 54.

The conclusion part of rule 9 assigned the fuzzy membership function 'high' to the output variable PD. Since the degree of truth of rule 9 when supplied with 30 and 54 was 0.85 , the output variable PD is assigned the following fuzzy subset when using PRODUCT inferencing:

$$
\operatorname{rule} 9(\mathrm{PD})=1.7 *(\mathrm{PD}-0.5)
$$

The membership graph is seen in Figure 12.

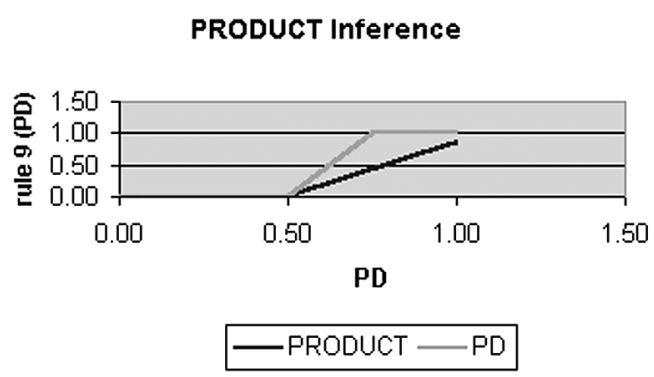

Fig. 12. The result using PRODUCT inference with rule9-TQ: 30 and WT: 54.

Notice, that the high membership function graph has been 'squashed down' so that the peak of the triangle measures 0.85 on the Y-axis.

\subsubsection{Composition}

Under COMPOSITION, all of the fuzzy subsets assigned to each output variable are combined together to form a single fuzzy subset for each output variable. Again, usually MAX or SUM are used. In MAX composition, the combined output fuzzy subset is constructed by taking the point wise maximum over all of the fuzzy subsets assigned to variable by the inference rule (fuzzy logic OR). In SUM composition, the combined output fuzzy subset is constructed by 
taking the point wise sum over all of the fuzzy subsets assigned to the output variable by the inference rule.

During the composition process, the fuzzy subsets assigned to the output variable are combined to form a single subset. Once again, there are two commonly used methods for fuzzy composition, namely MAX composition and SUM composition. Each will be discussed in turn.

When supplied with the input values 30 and 54 for TQ and WT, the results of the four rules were as follows:

rule1: 0.00

rule2: 0.00

rule3: 0.00

rule4: 0.00

rule5: 0.00

rule6: 0.00

rule 7: 0.15 'fire'

rule8: 0.30 'fire'

rule9: 0.85 'fire'

From these results we were able to use MIN inferencing to derive the following membership functions:

rule $1(\mathrm{PD})=0.00$

rule $2(\mathrm{PD})=0.00$

rule $3(\mathrm{PD})=0.00$

rule $4(\mathrm{PD})=0.00$

rule $5(\mathrm{PD})=0.00$

rule $6(\mathrm{PD})=0.00$

$\begin{array}{cl}\text { rule7 } \\ (\mathrm{PD})\end{array}=\left\{\begin{array}{cl}0.00, & \text { if } \mathrm{PD} \leq 0.25 \\ & \text { or } \mathrm{PD} \geq 0.75 \\ 0.15, & \text { if } \mathrm{PD}=0.50 \\ 0.6 *(\mathrm{PD}-0.25), & \text { if } 0.25<\mathrm{PD}<0.50 \\ 0.15-(0.6 *(\mathrm{PD}-0.50)), & \text { if } 0.50<\mathrm{PD}<0.75\end{array}\right.$

rule8 $(\mathrm{PD})=\left\{\begin{array}{cl}0.00, & \text { if } \mathrm{PD} \leq 0.50 \\ 6 *(\mathrm{PD}-0.50), & \text { if } 0.50 \leq \mathrm{PD} \leq 0.57 \\ 0.30, & \text { if } \mathrm{PD} \geq 0.57\end{array}\right.$

rule9 $(\mathrm{PD})=\left\{\begin{array}{cl}0.00, & \text { if } \mathrm{PD} \leq 0.50 \\ 2.428 *(\mathrm{PD}-0.5), & \text { if } 0.5 \leq \mathrm{PD} \leq 0.85 \\ 0.85, & \text { if } \mathrm{PD} \geq 0.85\end{array}\right.$

In MAX composition, the combined output fuzzy subset is constructed by taking the point wise maximum over all of the fuzzy subsets assigned to the output variable by the inference rule:

$$
\text { fuzzy }(\mathrm{PD})=\left\{\begin{array}{cl}
0, & \text { if } \mathrm{PD} \leq 0.25 \\
0.6 *(\mathrm{PD}-0.25), & \text { if } 0.25<\mathrm{PD}<0.50, \\
0.15-(0.6 *(\mathrm{PD}-0.50)), & \text { if } 0.50<\mathrm{PD}<0.52, \\
0.6 *(\mathrm{PD}-0.50), & \text { if } 0.52 \leq \mathrm{PD} \leq 0.57, \\
03, & \text { if } 0.57 \leq \mathrm{PD} \leq 0.62, \\
2.428 *(\mathrm{PD}-0.5), & \text { if } 0.62 \leq \mathrm{PD} \leq 0.85, \\
0.85, & \text { if } \mathrm{PD} \geq 0.85
\end{array}\right.
$$

This gives us the following membership graph (Figure 13).

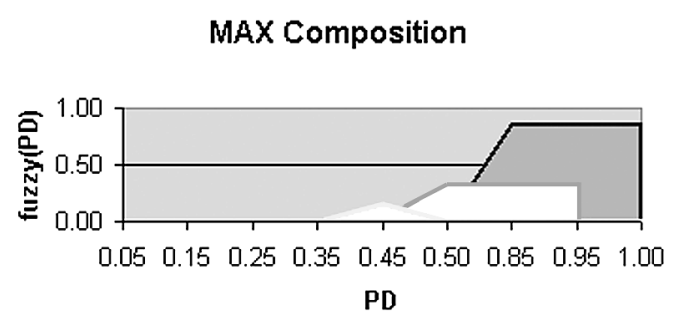

Fig. 13. MAX Composition.

The result with Traffic Quantity 30 and Waiting Time 54 is shown in Figure 14:

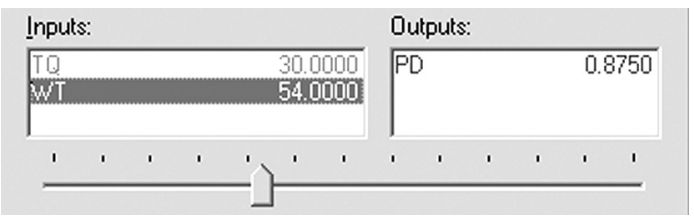

Fig. 14. Truth-value of Priority Degree.

From the rule alphas, we were also able to use PRODUCT inferencing to derive the following membership functions:

$$
\begin{aligned}
& \text { rule } 1(\mathrm{PD})=0.00 \\
& \text { rule } 2(\mathrm{PD})=0.00 \\
& \text { rule3 }(\mathrm{PD})=0.00 \\
& \text { rule } 4(\mathrm{PD})=0.00 \\
& \text { rule5 }(\mathrm{PD})=0.00 \\
& \text { rule6 }(\mathrm{PD})=0.00 \\
& \text { rule7.a }(\mathrm{PD})=0.6 *(\mathrm{PD}-0.25) \\
& \text { rule7.b }(\mathrm{PD})=0.15-(0.6 *(\mathrm{PD}-0.50)) \\
& \text { rule8 }(\mathrm{PD})=0.6 *(\mathrm{PD}-0.5) \\
& \text { rule } 9(\mathrm{PD})=1.7 *(\mathrm{PD}-0.5)
\end{aligned}
$$

SUM composition of the combined output fuzzy subset is constructed by taking the point wise sum over all of the fuzzy subsets assigned to the output variable by the inference rule: 


$$
\begin{aligned}
\text { fuzzy }(\mathrm{PD})= & 0.6 * \mathrm{PD}-0.15+0.15-(0.6 * \mathrm{PD}-0.3) \\
& +0.6 * \mathrm{PD}-0.3+1.7 * \mathrm{PD}-0.85 \\
= & 0.6 * \mathrm{PD}-0.15+0.15-0.6 * \mathrm{PD}+0.15 \\
& +0.3+0.6 * \mathrm{PD}-0.3+1.7 * \mathrm{PD}-0.85 \\
= & 2.3 * \mathrm{PD}-0.7
\end{aligned}
$$

This gives us the membership graph shown in Figure 15. Sometimes it is useful to just examine the fuzzy subsets that are the result of the composition process, but more often, this fuzzy value needs to be converted to a single number - a crisp value. This is what the defuzzification sub-process does.

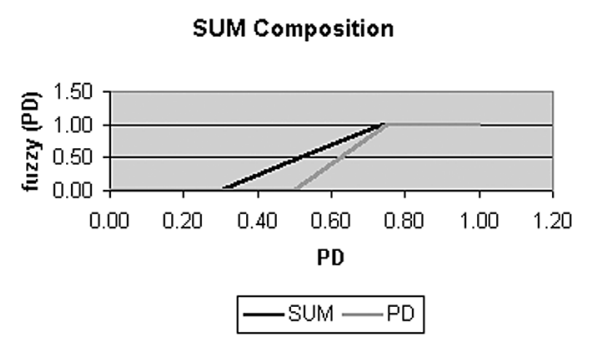

Fig. 15. SUM Composition.

\subsubsection{Defuzzification}

Finally, (optional) DEFUZZIFICATION is used when it is useful to convert the fuzzy output set to a crisp number. There are many defuzzification methods (at least 30). Two of the more common techniques are the CENTROID and MAXIMUM methods. In the CENTROID method, the crisp value of the output variable is computed by finding the variable value of the center of gravity of the membership function for the fuzzy value. In the MAXIMUM method, one of the variable values at which the fuzzy subset has its maximum truth-value is chosen as the crisp value for the output variable.

Applying the MAXIMUM method, the output truth value is:

$\operatorname{rule}(\mathrm{PD})=\operatorname{rule} 9(\mathrm{PD})=1.7 *(\mathrm{PD}-0.5)$

\section{System Analysis and Discussion}

This section presents a case study for a roundabout to verify the whole project. This is to verify manual results against the automated results obtained in practice.

\subsection{Traffic Control System Function}

Assume four incoming lanes for a roundabout with the following Traffic Quantity - TQ and Waiting Time $-\mathrm{WT}$ readings. These two inputs will be detected for each incoming lane.

\begin{tabular}{|c||c|c|c|c|}
\hline & Lane 1 & Lane 2 & Lane 3 & Lane 4 \\
\hline \hline TQ & 30 & 6 & 2 & 13 \\
\hline WT & 54 & 41 & 4 & 30 \\
\hline Rule1 & - & 0.47 & 1.00 & 0.20 \\
\hline Rule2 & - & 0.90 & - & 0.20 \\
\hline Rule3 & - & 0.53 & - & 0.20 \\
\hline Rule4 & - & 0.20 & - & 0.40 \\
\hline Rule5 & - & 0.20 & - & 0.40 \\
\hline Rule6 & - & 0.20 & - & 0.25 \\
\hline Rule7 & 0.15 & 0.10 & - & 0.75 \\
\hline Rule8 & 0.30 & 0.10 & - & 0.50 \\
\hline Rule9 & 0.85 & 0.10 & - & 0.25 \\
\hline
\end{tabular}

Table 6. Incoming lanes readings and their truth-values.

The result with Traffic Quantity 30 and Waiting Time 54 is shown in Figure 16 and graphical representation is shown in Figure 17:

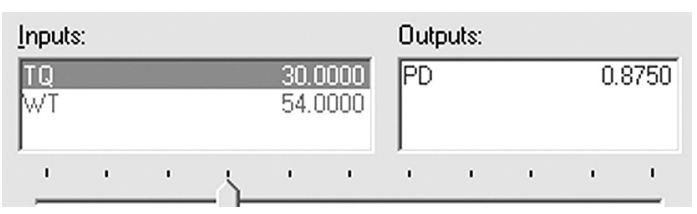

Fig. 16. Truth-value of Priority Degree.

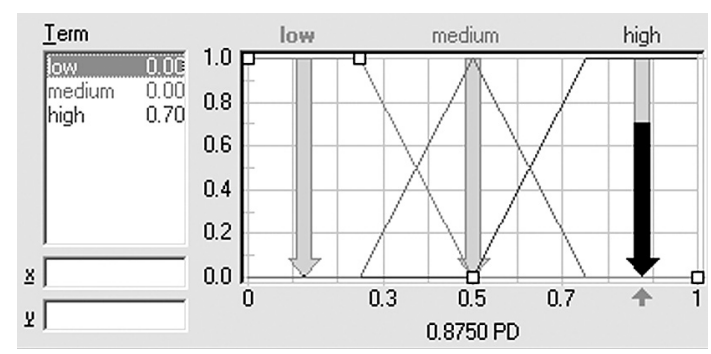

Fig. 17. Graphical representation of truth-value.

The result with Traffic Quantity 6 and Waiting Time 41 is shown in Figure 18, and graphical representation is shown in Figure 19: 


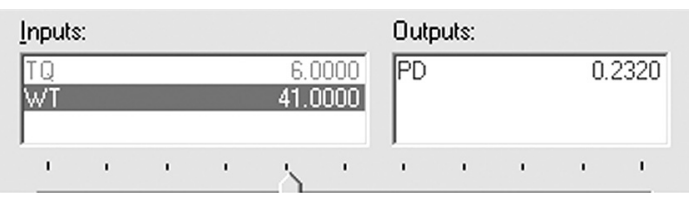

Fig. 18. Truth-value of Priority Degree.

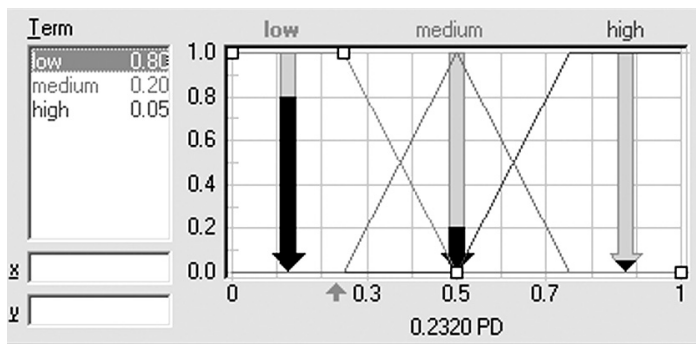

Fig. 19. Graphical representation of truth-value.

The result with Traffic Quantity 2 and Waiting Time 4 is shown in Figure 20 and graphical representation is shown in Figure 21:

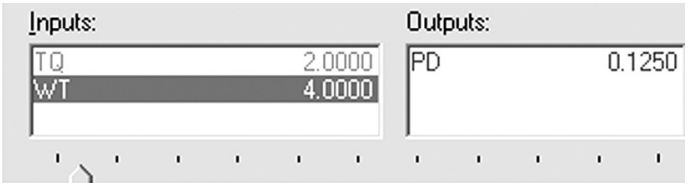

Fig. 20. Truth-value of Priority Degree.

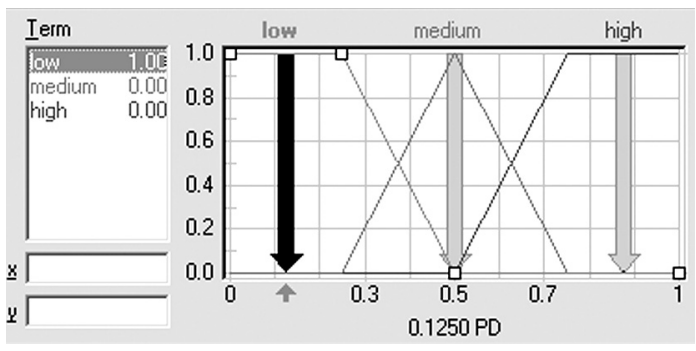

Fig. 21. Graphical representation of truth-value.

The result with Traffic Quantity 13 and Waiting Time 30 is shown in Figure 22 and graphical representation is shown in Figure 23:

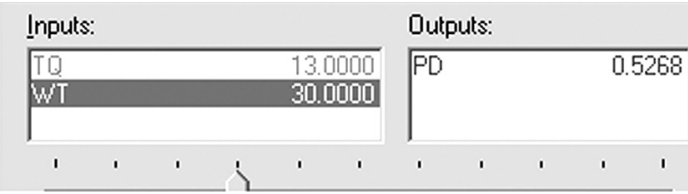

Fig. 22. Truth-value of Priority Degree.

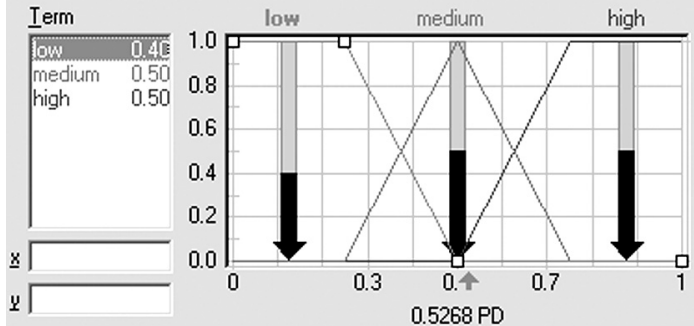

Fig. 23. Graphical representation of truth-value.

\subsection{Traffic Light Signals Lightening Function According to Inference Process Outcomes}

The priority degree variable for the four lanes is defined (calculated) according to rule 9 that measures the high(Traffic Quantity) and high (Waiting Time), so the approach with highest value of rule 9 wins and so on.

First: Lane one traffic signal will light green because it has the maximum value of rule 9 , when all cars pass, it turns red.

Second: Lane four lights green and the other three lanes traffic signals light red.

Third: Lane two lights green and the other three lanes signals light red.

Fourth: Lane three lights green, although it does not 'fire' for rule 9, but the lane 'fires' at rule 1 , which means there are cars waiting in the lane.

\section{Conclusion}

The fuzzy logic traffic light controller is performing better than the fixed-time controller, or even vehicle-actuated controllers. The reason is that it counts the number of vehicles sensed at the incoming approaches and their waiting time then finds the winning approach for green light. With the fixed-time controller, the green light time is not extended whatever the density of cars at the approach. For vehicle-actuated traffic light controllers, which are an enhanced version of fixed-time controller, the green light time is extended whenever there is a vehicle. However, these times are fixed prior to maximum time limit. In the fuzzy logic controller, 
the extension time is not a fixed value. The number of cars sensed at the input of the fuzzy controllers are converted into fuzzy values, such as low, medium, high, etc. In addition to the fuzzy variables, the fuzzy controller also has the advantage of performing according to linguistic rules in the manner of how a human would. The inferencing method in the fuzzy controller is similar to the way a traffic policeman handles the traffic flow at a typical roundabout. A comparison was made between the performance of the fuzzy logic controller and that of a fixedtime conventional controller. It can be observed from the results that the fuzzy logic control system provides better performance in terms of total waiting time as well as of total moving time. Less waiting time will not only reduce the fuel consumption, but it will also reduce air pollution and noise. Finally, the proposed adaptive traffic system will provide consistency and independency in decision making more than any other traffic systems. Future work is to investigate if the controller performance is to be improved by incorporating in the model the density of traffic in the nearest intersections.

\section{References}

[1] I. GRAHAM AND P. L. JONES, Expert Systems Knowledge: Uncertainty and Decision, Chapman and Hall, London and New York, pp. 117-118, 1988.

[2] A. Hegyi, B. De Schutter And S. HoOgendoorn, Fuzzy Decision Support System for Traffic Control Centers, Paper presented to the Delft University of Technology, at Delft, Netherlands, 1999.

[3] C. TAYlor, D. Meldrum, Fuzzy Ramp Implementation, Research Project prepared by Department of Electrical Engineering of University of Washington, at Seattle, Washington 98195, pp. 1,6, 2000.

[4] I. DAY, SCOOT- Split, Cycle \& Offset Optimization Technique, presented by Siemens AG, Traffic Control Systems Division, presented to Transportation Research Board, TRB Committee A3A18 Traffic Signal Systems, TRB Mid- Year Meeting and Adaptive Traffic Signal Control Workshop, pp. 6,9,12,13, 1998.

[5] J. NiITTYMÄKI AND M. MAEnPäÄ, Fuzzy Public Transport Priority in Traffic Signal Control, Paper presented to Helsinki University of Technology, Transportation Engineering, at Hut, Finland, 2000.

[6] W. Wei And M. WANG, Traffic Signal Control Using Fuzzy And Neural Network, Paper presented to Department of Road and Traffic Engineering, Changsha Communications University, Changsha, 410076, P.R. China, 2000.
Received: October, 2005

Revised: March, 2006

Accepted: March, 2006

Contact address:

Maged M. M. Fahmy

College of Applied Studies King Faisal University Saudi Arabia

Postal Code 31952 P.O.B. 40287

Al Khobar, KSA

e-mail: dr_maged_fahmy@yahoo.com

MAGED M. M. FAHMY is currently working as assistant professor in the College of Applied Studies, King Faisal University, Saudi Arabia. From 1999-2005 he worked as assistant professor in the Computer Science Department of the Information Technology College, University of Bahrain. From 1995 to 1999 , he was engaged in research at the Informatics Research Institute in Mubarak City, for Scientific Research and Technological Application, Alexandria, Egypt. Parallelly he was acting as assistant professor at the Faculty of Science, Computer Division, Alexandria University. He got B.Sc. in Electronic Engineering and M.Sc. in Computer Security from Alexandria University in 1986. $\mathrm{He}$ got $\mathrm{Ph}$.D. in Engineering in Computer Vision from University of Newcastle (U.K) in 1995. He supervised many Master theses in both of Faculty of Engineering \& Faculty of Science, Alexandria University. His fields of interest are programming languages, artificial intelligence, CTI applications, computer vision, fuzzy logics, and computer networks. 